\title{
Artemisinin and its derivatives can significantly inhibit lung tumorigenesis and tumor metastasis through $\mathrm{Wnt} / \beta$-catenin signaling
}

\author{
Yunli Tong ${ }^{1,2, *}$, Yuting Liu ${ }^{1,2, *}$, Hongming Zheng ${ }^{2}$, Liang Zheng ${ }^{1,2}$, Wenqin Liu ${ }^{1,2}$, \\ Jinjun $\mathrm{Wu}^{2}$, Rilan $\mathrm{Ou}^{2}$, Guiyu Zhang ${ }^{2}$, Fangyuan $\mathrm{Li}^{2}$, Ming $\mathrm{Hu}^{2,3}$, Zhongqiu Liu ${ }^{1,2}$, \\ Linlin Lu ${ }^{2}$ \\ ${ }^{1}$ School of Pharmaceutical Sciences, Southern Medical University, Guangzhou, Guangdong, 510515, China \\ ${ }^{2}$ International Institute for Translational Chinese Medicine, Guangzhou University of Chinese Medicine, Guangzhou, \\ Guangdong, 510006, China \\ ${ }^{3}$ Department of Pharmacological and Pharmaceutical Sciences, College of Pharmacy, University of Houston, Houston, Texas, \\ 77030, USA \\ *These authors have contributed equally to this work \\ Correspondence to: Zhongqiu Liu, e-mail: liuzq@gzucm.edu.cn, liuzq@smu.edu.cn \\ Linlin Lu, e-mail: Illu@gzucm.edu.cn \\ Keywords: non-small-cell lung cancer, artemisinin, derivative, Wnt/ $\beta$-catenin \\ Received: November 16, 2015 \\ Accepted: April 02, 2016 \\ Published: April 22, 2016
}

\section{ABSTRACT}

Non-small-cell lung cancer (NSCLC) is the most prevalent malignancy worldwide given its high incidence, considerable mortality, and poor prognosis. The anti-malaria compounds artemisinin (ART), dihydroartemisinin (DHA), and artesunate (ARTS) reportedly have anti-cancer potential, although the underlying mechanisms remain unclear. In this work, we used flow cytometry to show that ART, DHA, and ARTS could inhibit the proliferation of A549 and H1299 cells by arresting cell cycle in G1 phase. Meanwhile, tumor malignancy including migration, invasion, cancer stem cells, and epithelial-mesenchymal transition were also significantly suppressed by these compounds. Furthermore, ART, DHA, and ARTS remarkably decreased tumor growth in vivo. By using IWP-2, the inhibitor of Wnt/ $\beta$-catenin pathway, and Wnt5a siRNA, we found that ART, DHA, and ARTS could render tumor inhibition partially dependent on Wnt/ $\beta$-catenin inactivation. These compounds could strikingly decrease the protein level of Wnt5-a/b and simultaneously increase those of NKD2 and Axin2, ultimately resulting in $\beta$-catenin downregulation. In summary, our findings revealed that ART, DHA, and ARTS could suppress lung-tumor progression by inhibiting Wnt/ $\beta$-catenin pathway, thereby suggesting a novel target for ART, DHA, and ARTS in cancer treatment.

\section{INTRODUCTION}

Lung cancer remains the leading cause of cancerrelated deaths worldwide because of its high incidence and mortality $[1,2]$. Non-small-cell lung cancer (NSCLC), which accounts for approximately $85 \%$ of all lung cancer cases, is composed of various histological subtypes, among which adenocarcinomas are responsible for almost $40 \%$ diagnoses $[3,4]$. Given that $67.6 \%$ of lungcancer patients have been diagnosed in advanced stages, chemotherapies including molecular-drug-targeted mutations of epidermal growth factor receptor (EGFR) remain the best and standard strategies for lung cancer treatments after surgery. However, drug resistance, subsequent metastasis, and accumulated toxicity to normal cell and immune system limit the clinical benefit of chemotherapies and the median survival to 1 year [4, 5]. Therefore, utilizing food-derived, plant-derived, or pharmaceutical interventions with broad effectiveness and tolerable side effects to preventtumor malignancy and invasion is a promising approach for metastasis reduction, and overall survival improvement [6]. 
Wnt/ $\beta$-catenin signaling pathway, prominently activated in NSCLC, plays a critical role in lung tumorgenesis and metastasis and also mediates drug resistance [7]. Wnt5-a/b, defined as "destruction complex" along with Frizzled receptors, lipoprotein-receptorrelated protein (LRP), dishevelled (Dvl), and scaffold protein axis inhibition protein (Axin), is a prerequisite for Wnt/ $\beta$-catenin activation. Conversely, Axin2 [8] and Drosophila naked cuticle (NKD2) [9], which are negative regulators in canonical Wnt signaling, could suppress $\beta$-catenin by inhibiting its transcription while promoting ubiquitination [10]. With increased $\beta$-catenin translocated from cytoplasma to nucleus [11, 12], the transcriptional activities of various downstream genes including cyclin D1, oct3/4, sox 2, and nanog are strikingly stimulated [1315]. The abundant expressions of sox 2 , oct $3 / 4$, and nanog represent the pluripotency of cancer stem cells (CSCs) $[14,16]$, and cyclin D1 serves as a primary mediator in cell cycle maintenance [17]. Hence, the activation of $\mathrm{Wnt} / \beta$-catenin may result in increased malignancy and continuous tumor cell proliferation. Moreover, by decreasing the protein expression of E-cadherin, while increasing that of N-cadherin and vimentin, epithelialmesenchymal transition (EMT) could also be promoted by $\beta$-catenin in lung cancer metastasis [18]. The schematic of Wnt/ $\beta$-catenin pathway is shown in Figure 7.

Some epidemiological studies have revealed that various dietary compounds such as green tea catechins, lycopene, selenium, and vitamin A could be regard as chemopreventive agents for lung cancer recurrence and metastasis [6]. Especially, some phytochemical agents including resveratrol [19], curcumin [20], and wogonin [21] also reportedly have anti-tumor efficacy by suppressing $\mathrm{Wnt} / \beta$-catenin pathway. However, partially due to low pulmonary bioavailability and unclear targets of these alternative therapeutic agents, clinical trials for NSCLC chemoprevention have yielded negative results $[22,23]$. Thus, identifying a component with better physicochemical property, less toxicity, and certain molecular targets can aid research on lung cancer treatment and survival improvement.

Artemisia anпиа L., a traditional medicine used for more than 2000 years, has significant efficacy against malaria, parasitic diseases, and fibrosis with low toxicity [24]. Artemisinin (ART), along with its derivatives such as artesunate (ARTS) and dihydroartemisinin (DHA), are the safe, predominant compounds in anti-malarial treatment [25-28]. Meanwhile, the conventional administration of ART, DHA, and ARTS depends on oral delivery, suggesting the high relative bioavailability of these agents [29]. According to some pharmacokinetic studies, the strong amphipathic characteristics of ARTS and DHA lead to the clearance time of $6 \mathrm{~h}$ after a single intravenous injection [30]. Among three compounds, DHA had the highest relative bioavailability $(>80 \%)$ after oral intake in rats and humans [31, 32].
Recent studies have revealed that these three components markedly inhibit tumor growth and metastasis of NSCLC with preferential cytotoxicity in tumor cells even in micromoles [33-36]. Other studies have demonstrated that DHA induced lung cancer cell apoptosis by inhibiting the phosphorylation of p70 S6 kinase 1 in an mTORC1-dependent manner [37]. Also reports have shown that ART notably suppressed tumor invasion and metastasis in NSCLC cells. Whereas whether ART, DHA, and ARTS could suppress tumors by inhibiting Wnt/ $\beta$ catenin pathway, as well as the precise molecular targets involved, remains unclear. Additionally, the potential of these herbal medicines with low toxicity and high anti-tumor effectiveness to become chemopreventive candidates is under investigation.

In the current study, by utilizing an A549 xenograft model, the anti-tumor efficacy of ART, DHA, and ARTS were addressed in vivo. In addition, the anti-proliferation and anti-metastasis effects of ART and its two derivatives were further evaluated in two different lung cancer cell lines, including A549 (carcinoma in situ) and H1299 (adenocarcinoma from metastasis nodule). Furthermore, in $\mathrm{Wnt} / \beta$-catenin pathway, we also investigated the underlying mechanism and explored drug-sensitive targets. Considering the low cytotoxicity and high bioavailability of ART, DHA, and ARTS, determining the effect of these compounds on $\mathrm{Wnt} / \beta$-catenin pathway may provide information on their potential as perfect chemopreventive candidates.

\section{RESULTS}

\section{ART, DHA, and ARTS suppressed cell viability and induced G1 arrest by inhibiting cyclin D1 in A549 and $\mathrm{H} 1299$}

MTT and flow cytometry assays were conducted to investigate the effects of ART, DHA, and ARTS on the cell fate of A549 and H1299 cells. After treating cells with ART, DHA, and ARTS $(0-200 \mu \mathrm{M})$ for $48 \mathrm{~h}$, cell viabilities were significantly suppressed. Additionally, the sensitivities of tumor cell inhibition among these compounds decreased in the order DHA > ARTS > ART. However, ART, DHA, and ARTS treatments did not effectively inhibit the growth of BEAS-2B (normal human bronchial epithelial cells) up to $70 \mu \mathrm{M}$ because $\mathrm{IC}_{50}$ was observed only in DHA at $76.95 \mu \mathrm{M}$ (Figure 1A). Furthermore, with increased concentrations of ART, DHA, and ARTS, tumor cells arrested in G1 phase significantly accumulated. Especially, ART at $30 \mu \mathrm{M}$ markedly increased the percentage of tumor cells in G1 phase with $17.63 \% \pm 0.67 \%(p<0.001)$ and $26.69 \% \pm 0.68 \%(p<0.01)$ in A549 and H1299, respectively (Figure 1B). However, none of ART, DHA, and ARTS could notably alter the cell cycle of BEAS-2B (Supplementary Figure S1). Cyclin D1, the vital regulator of G1 progression, of which protein level was significantly 

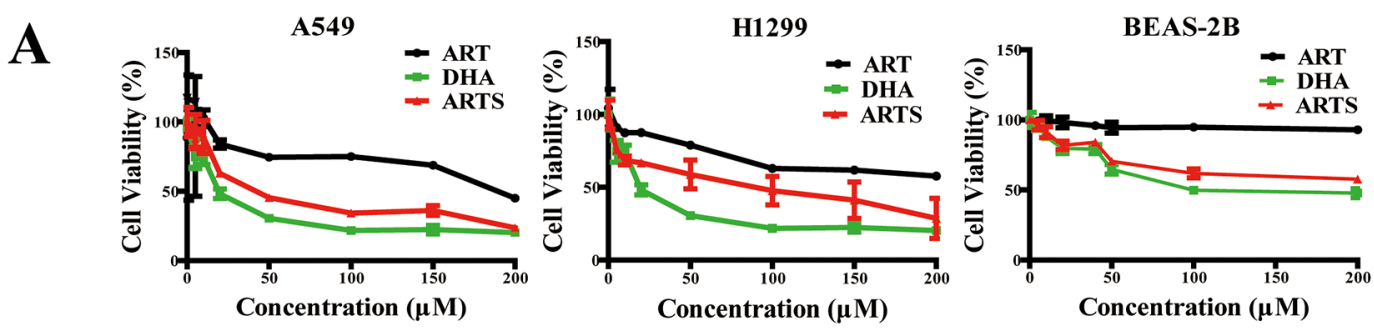

B
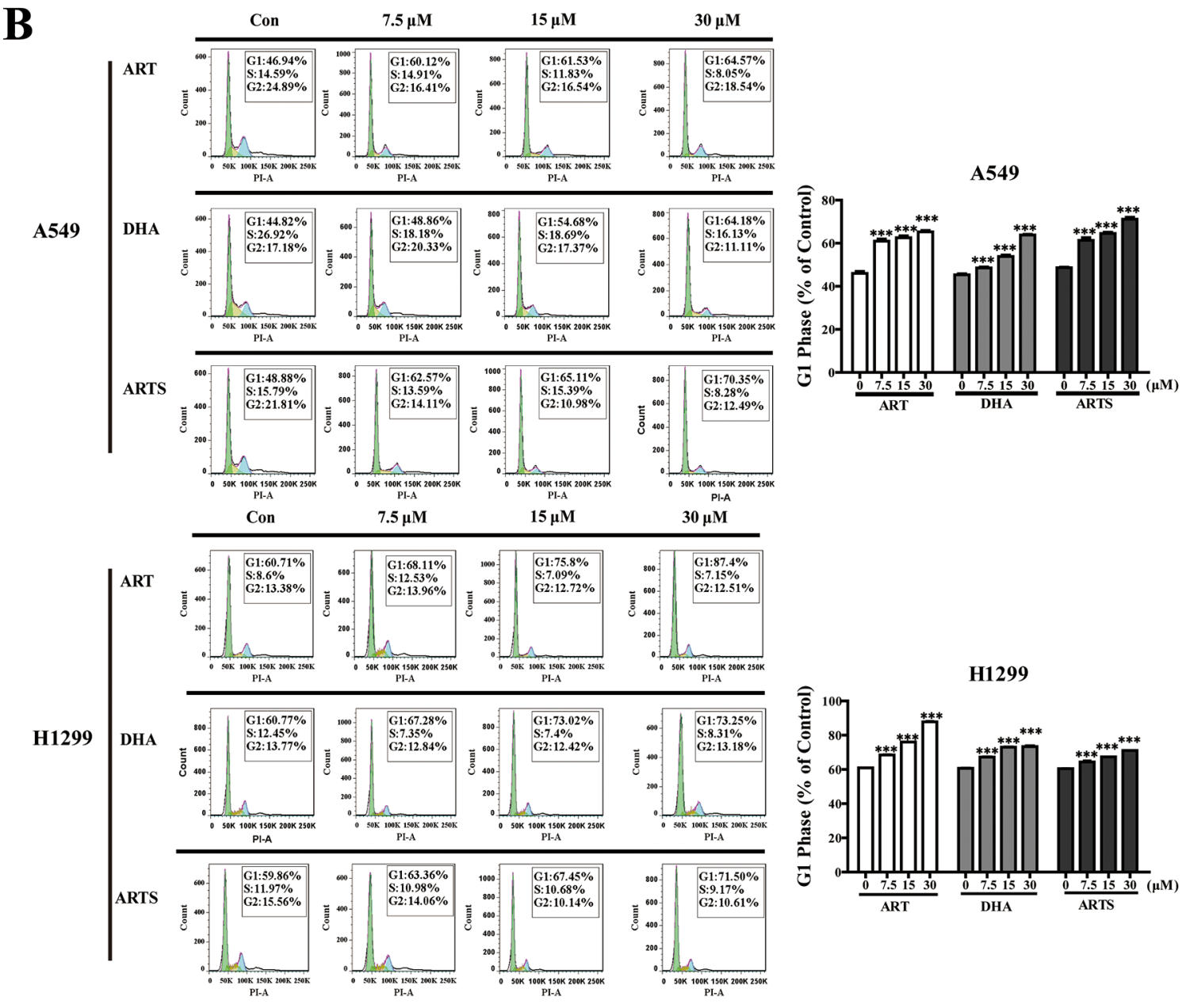

$\mathbf{C}$
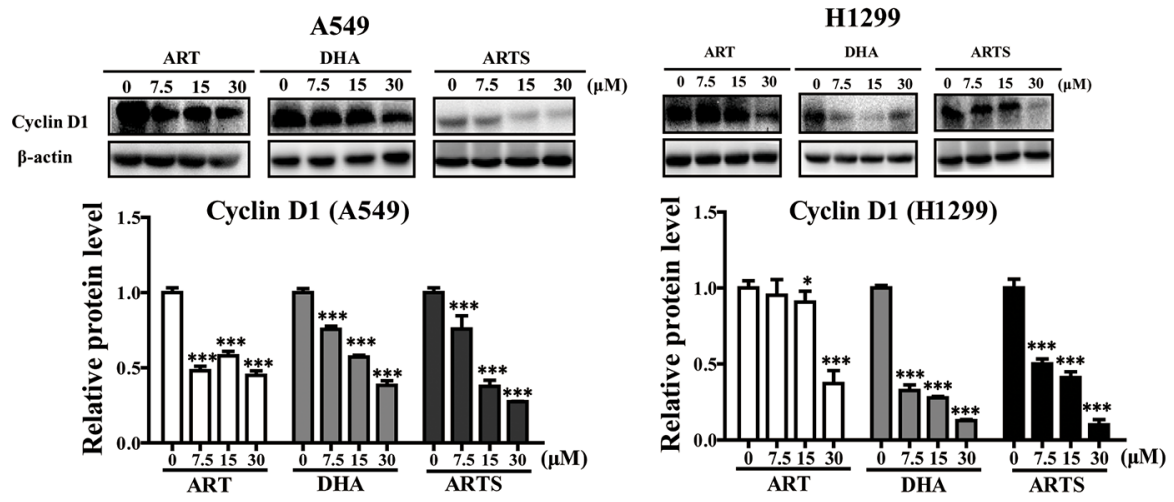

Figure 1: Effects of artemisinin (ART), dihydroartemisinin (DHA), and artesunate (ARTS) on cell viabilities and cell cycle progression. A. Relative cell viabilities of A549, H1299, and BEAS-2B treated with ART, DHA, and ARTS $(0-200 \mu \mathrm{M})$. B. Percentages of A549 and H1299 cells in the G1 phase after treatment with or without ART, DHA and ARTS. C. Effects of ART, DHA, and ARTS on expression of cyclin D1. Each column represented the mean \pm standard deviation (SD). ${ }^{*} p<0.05,{ }^{* * *} p<0.001$, compared with the control group (Con, control). 
reduced by ART, DHA, and ARTS both in A549 and H1299 cells. Yet, cyclin D1 decreased in a dose-dependent manner only in DHA and ARTS treatments. Additionally, ARTS also exerted greater suppressive effect on cyclin D1 expression than ART and DHA (Figure 1C).

\section{Cell invasion and migration were inhibited by ART, DHA, and ARTS}

Wound healing assay was used to evaluate migration in A549 and H1299 cells. ART, DHA, and ARTS significantly inhibited lung tumor cell migration in a dosedependent manner. Among all three compounds, DHA at $30 \mu \mathrm{M}$ showed the most effective migration suppression with $27.1 \% \pm 3.52 \%$ compared with the control group (Figure 2A; $p<0.001$ ). Additionally, transwell coated with matrigel was used to explore the invasive ability of lung tumor cells. Compared with abundant cells translocated to the underside of well in the control treatment, cell invasion activities were dose-dependently decreased by ART, DHA, and ARTS. Especially in ART treatments, cell invasion significantly decreased by $32.19 \% \pm 0.15 \%(p<0.001)$, $58.77 \% \pm 1.79 \%(p<0.001)$, and $67.25 \% \pm 0.22 \%$ $(p<0.001)$, respectively, with increased concentrations (7.5, 15, and $30 \mu \mathrm{M}$; Figure 2B). Meanwhile, matrix metalloproteinase (MMPs) activities were analyzed by MMPs activity assay kit to further determine the invasion capability of ART, DHA, and ARTS. All ART, DHA, and ARTS treatments could markedly inhibit MMPs activities in a dose-dependent manner (Figure 2C).

\section{ART, DHA, and ARTS suppressed EMT and CSCs}

CSCs and EMT are two key factors affecting tumor metastasis, including migration and invasion. Whether the anti-metastasis effects of ART, DHA, and ARTS are associated with these two aspects were analyzed by Western blotting. Results showed that the expression levels of CSC markers including nanog, sox2, and oct3/4, as well as EMTrelated proteins such as $\mathrm{N}$-cadherin and vimentin, were all significantly inhibited by ART, DHA, and ARTS treatments, whereas those of E-cadherin either in A549 (Figure 3) or H1299 cells (Supplementary Figure S2) increased. Among the three compounds, ART exerted the greatest inhibitory effects, especially in the expression of nanog and sox2 in A549 cells, and the percentages of decrease at $30 \mu \mathrm{M}$ were $65 \% \pm 0.26 \%(p<0.001)$ and $80 \% \pm 1.92 \%(p<0.001)$, respectively (Figure 3A). Similarly, the protein levels of N-cadherin and vimentin were remarkably downregulated by ART (Figure 3B). However, in H1299 cells, most increased E-cadherin expression and decreased CSCs markers were observed in ARTS treatments (Supplementary Figure S2B), indicating that the effective response of ART, DHA, and ARTS may slightly vary in tumor cells with different histological features.

\section{ART, DHA, and ARTS depressed Wnt/ק-catenin signaling pathway}

To determine whether $\mathrm{Wnt} / \beta$-catenin pathway was involved in the anti-tumor and anti-metastasis effects of ART and its two derivatives, key proteins in $\mathrm{Wnt} / \beta$-catenin pathway were analyzed by Western blotting. In both A549 and H1299 cells, most dramatic reduction were found on the expressions of Wnt5-a/b after ART, DHA, and ARTS treatments, even at $7.5 \mu \mathrm{M}$ (Figure 4A and 4B). Furthermore, the subsequent proteins in Wnt/ $\beta$-catenin signaling pathway were also altered remarkably by ART and its two derivatives. For example, LRP6 significantly decreased by $16.80 \% \pm 1.14 \%$ after DHA $(30 \mu \mathrm{M})$ treatment in A549 cells (Figure 4A; $p<0.001$ ), whereas the protein levels of Dvl2 were markedly reduced by ARTS at $15 \mu \mathrm{M}$ by $6.50 \% \pm 0.64 \%(p<0.001)$ and $3.58 \%$ $\pm 0.42 \%(p<0.001)$, respectively, in A549 and H1299 cells (Figure 4A and 4B). Moreover, in those cell lines, ART, DHA, and ARTS treatments significantly decreased the protein of $\beta$-catenin accumulated either in cytoplasm or nucleus (Figure 4C).

\section{Suppression of ART, DHA, and ARTS in proliferation and metastasis could partially depend on Wnt/ $\beta$-catenin pathway inactivation}

To determine whether the anti-tumor effects of ART and its derivatives depended on Wnt/ $\beta$-catenin pathway, IWP-2, Wnt/ $\beta$-catenin pathway inhibitor and Wnt5a siRNA were used considering the important role of Wnt5$\mathrm{a} / \mathrm{b}$ and of which most protein reduction were observed previously. The expressions of $\mathrm{Wnt} 5-\mathrm{a} / \mathrm{b}$ and $\beta$-catenin in A549 cells were dramatically suppressed by IWP-2, whereas those of NKD2 and Axin2 were not altered (Figure 5A). However, pretreated with IWP-2, the G1 arrest induced by ART, DHA, and ARTS were enhanced in A549 cells. While interestingly, compared with IWP-2 treatment, no significant differences were observed in treatments of ART and its derivatives combined with IWP-2 (Figure 5B). Moreover, as expected, Wnt5$\mathrm{a} / \mathrm{b}$ knockdown consequently suppressed the protein expressions of sox 2 and $\beta$-catenin but increased that of E-cadherin. However, with Wnt5a knockdown, ART, DHA, and ARTS still exerted anti-metastasis effect by decreasing sox2 while increasing E-cadherin (Figure 5C). Therefore, with or without Wnt5a, the irreplaceable and sustained suppression of $\mathrm{Wnt} / \beta$-catenin pathway occurred, suggesting that the anti-tumor effects of ART and its derivatives only partially depended on Wnt5-a/b inactivation. On the other hand, Axin2 and NDK2, two negative regulators in canonical $\mathrm{Wnt} / \beta$-catenin signaling pathway, were simultaneously explored. In A549 cells, ART at $7.5,15$, and $30 \mu \mathrm{M}$ significantly increased the protein level of NKD2 by $132.38 \% \pm 4.60 \%(p<0.001)$, $198.83 \% \pm 12.25 \%(p<0.001)$, and $734.09 \% \pm 20.14 \%$ 


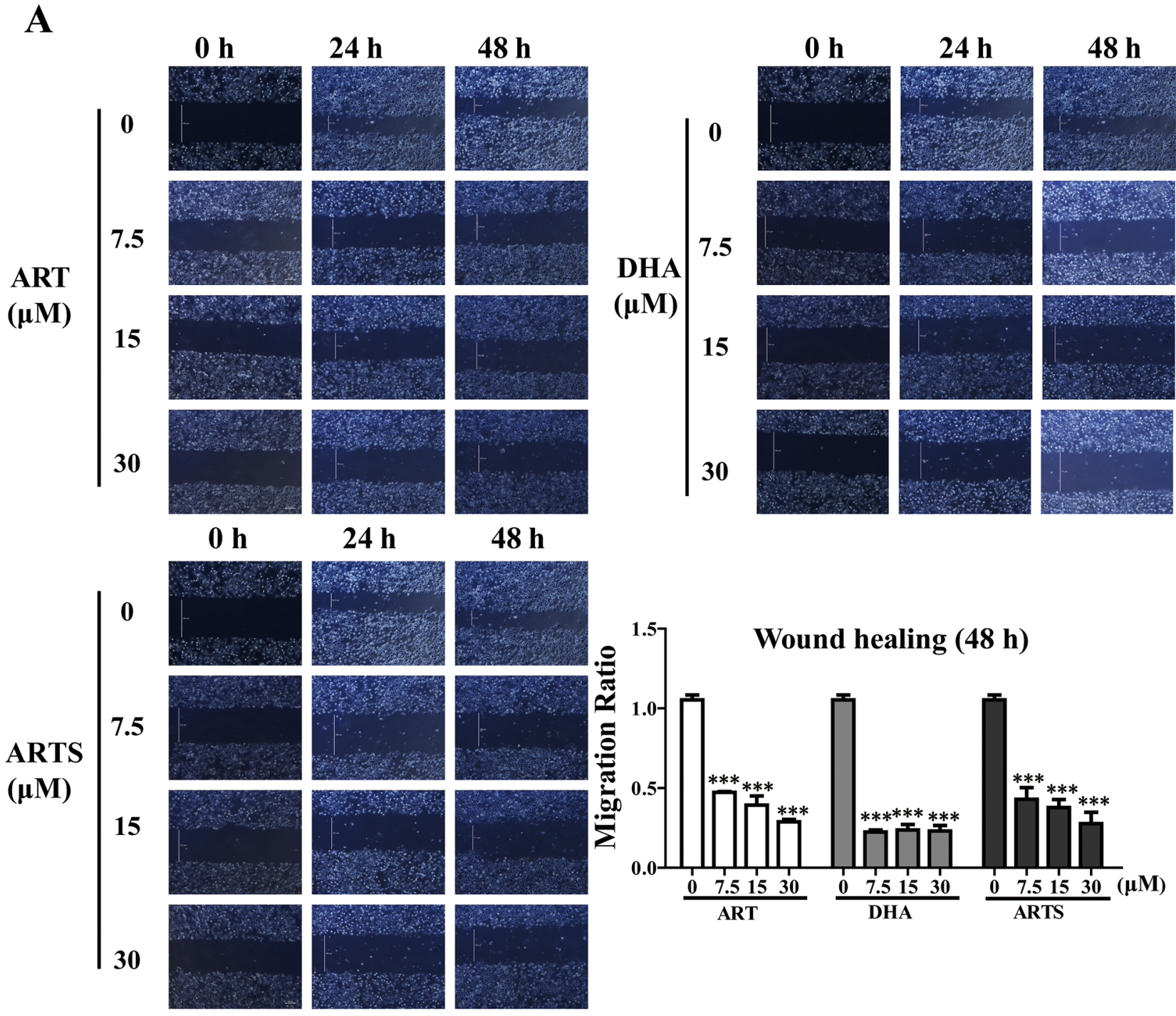

B
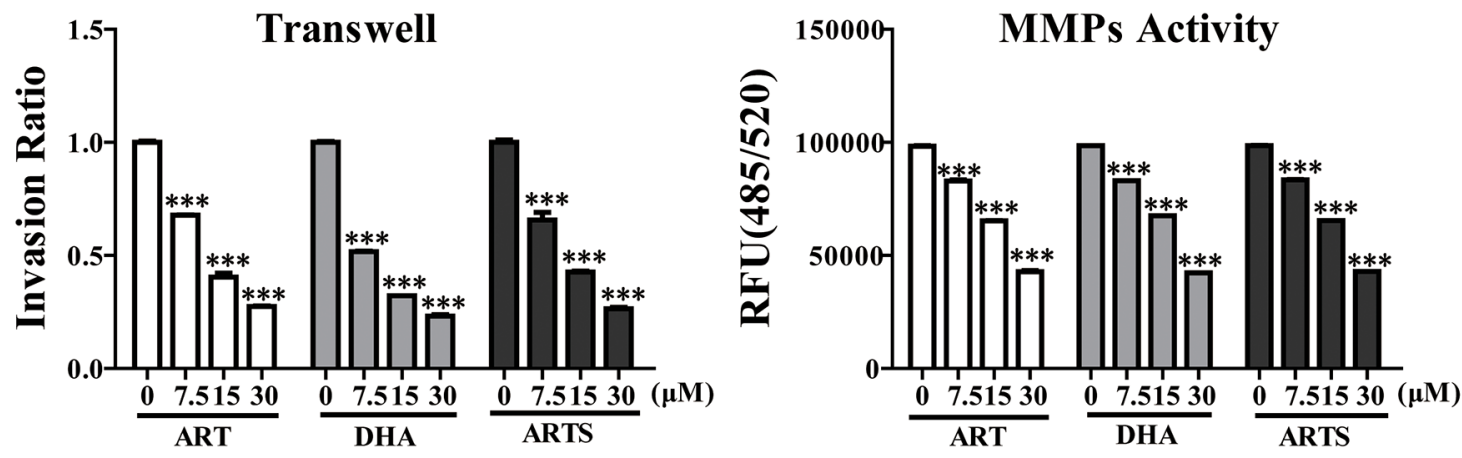

Figure 2: Effects of ART, DHA, and ARTS on cell migration and invasion. A. Representative photomicrographs of initial and final wounds at 24 and $48 \mathrm{~h}$ were shown at 100× magnification. Cell migration distance data were presented as the means \pm SD of three independent experiments. B. Cell invasion data in A549 cell line shown were the means \pm SD. C. Inhibitory effects of ART, DHA, and ARTS on MMPs activities were shown. Each bar was representative of the mean $\pm \mathrm{SD}$. $(* * * p<0.001$, compared with the control group). 
$(p<0.001)$, respectively (Figure 5D). Similar results were observed in H1299 cells (Figure 5E). Additionally, the expressions of Axin 2 were also notably increased by ART in A549 cells and by ARTS in H1299 cells.

\section{ART, DHA, and ARTS restrained lung cancer growth in A549 xenograft mice}

The A549 xenograft model was employed to further explore the effects and toxicity of ART, DHA, and ARTS in vivo. ART, DHA, and ARTS, as well as afatinib, did not cause significant body weight loss in mice (Figure 6A). While the tumor volumes also remarkably shrank after ART, DHA, and ARTS gavage, and maximum inhibition (41.04\%) was observed after ARTS treatment (Figure 6B; $p<0.01$ ). In addition, tumor weights were significantly reduced as well in the ART, DHA, and ARTS groups compared with that in the control group (Figure 6B; $p<0.05$ ).

To verify the observation in vitro, EMT and CSC markers and Wnt associated proteins were examined in tumor tissues from xenograft mice. Immunostaining

A

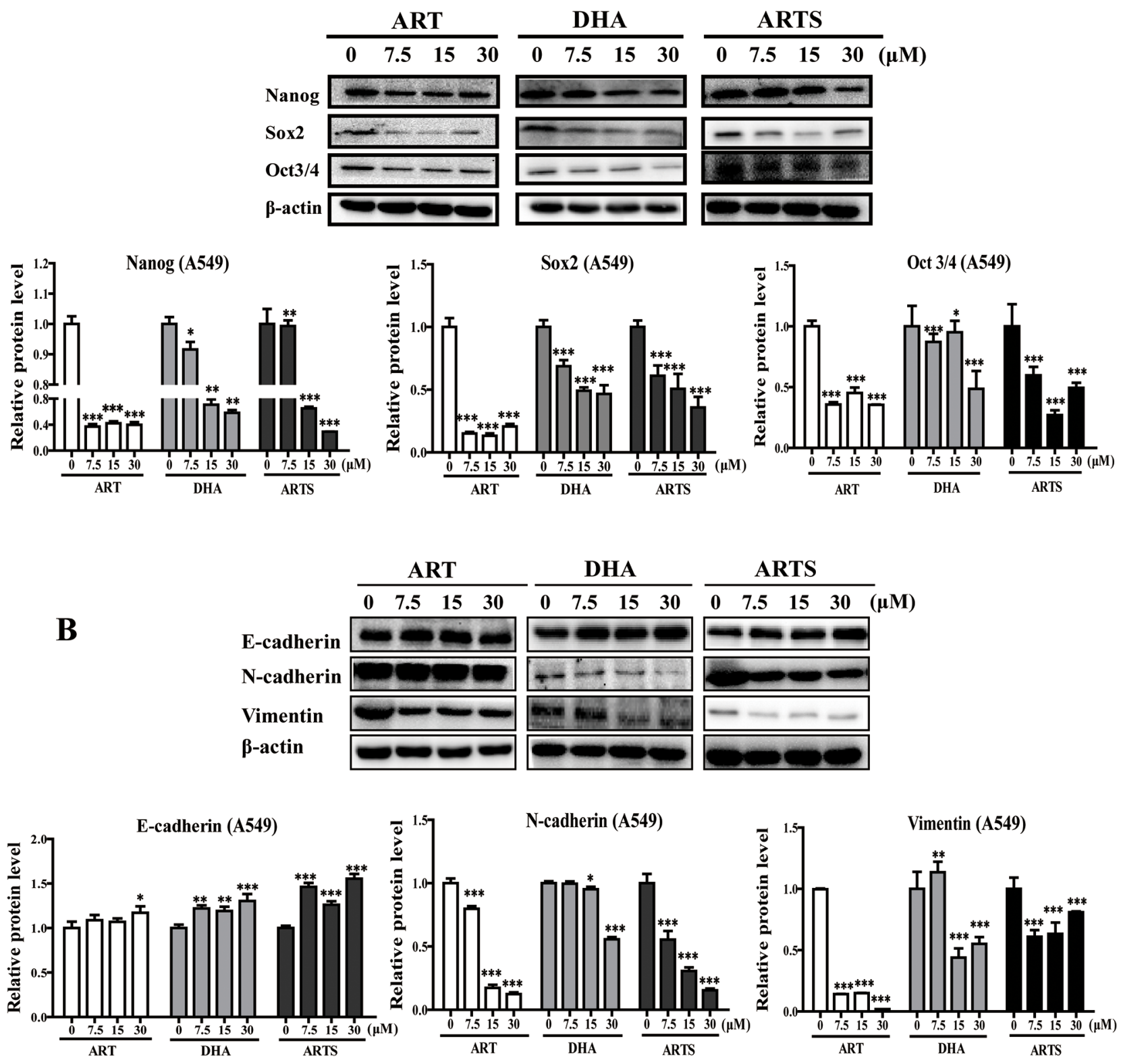

Figure 3: Epithelial-mesenchymal transition (EMT) and cancer stem cells (CSCs) were regulated by ART, DHA, and ARTS in A549 in vitro. A. Expression of CSCs regulatory proteins was determined by Western blot in A549 with antibodies against nanog, sox2, and oct3/4. B. Western blot analysis was performed for E-cadherin, N-cadherin, and vimentin. Data shown were the means \pm SD. $* p<0.05, * * p<0.01, * * * p<0.001$, compared with the control group (Con, control). 


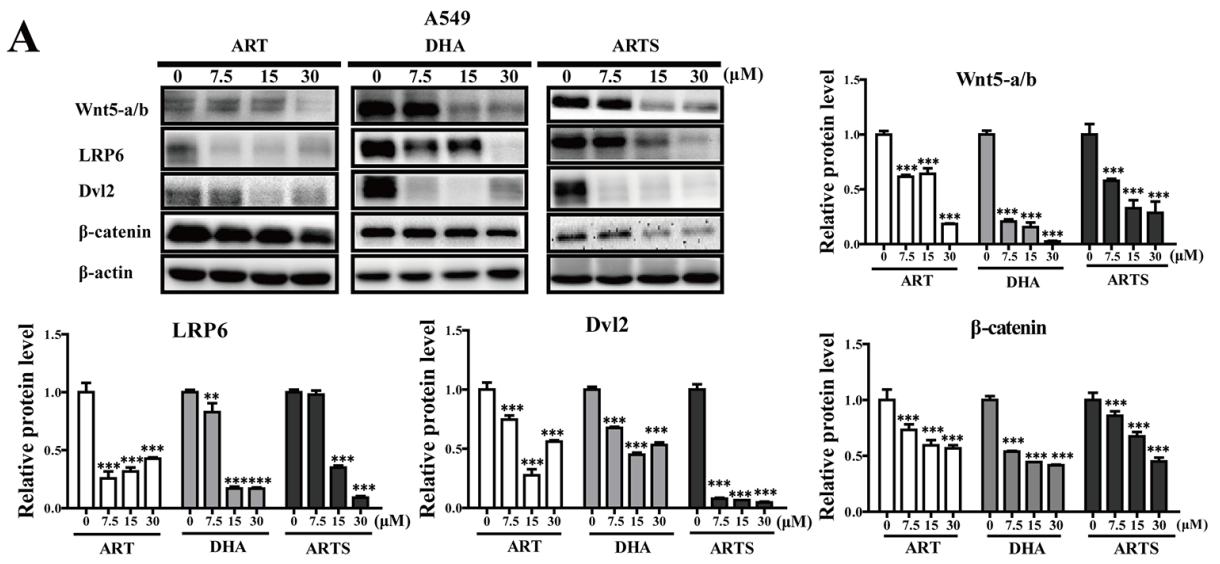

B
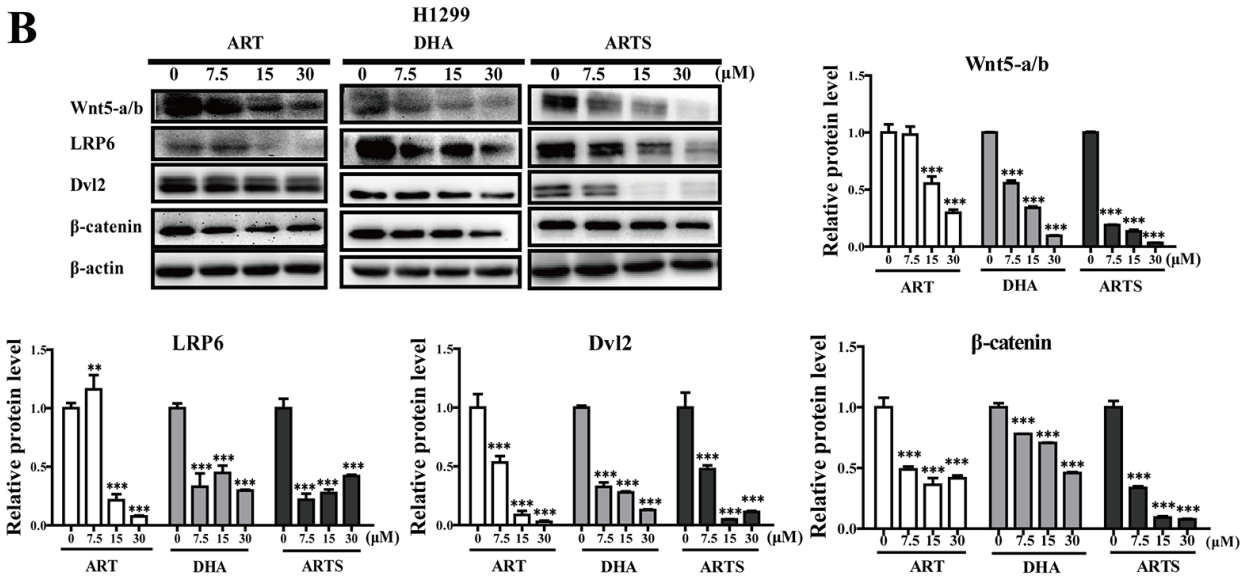

C

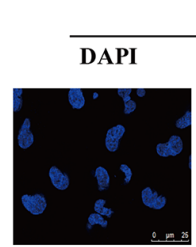

A549
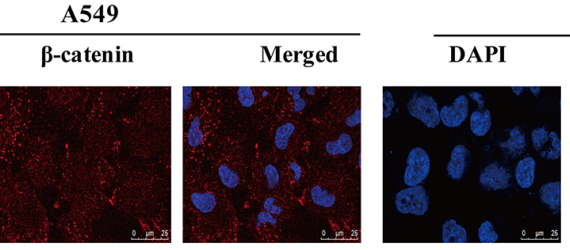

H1299
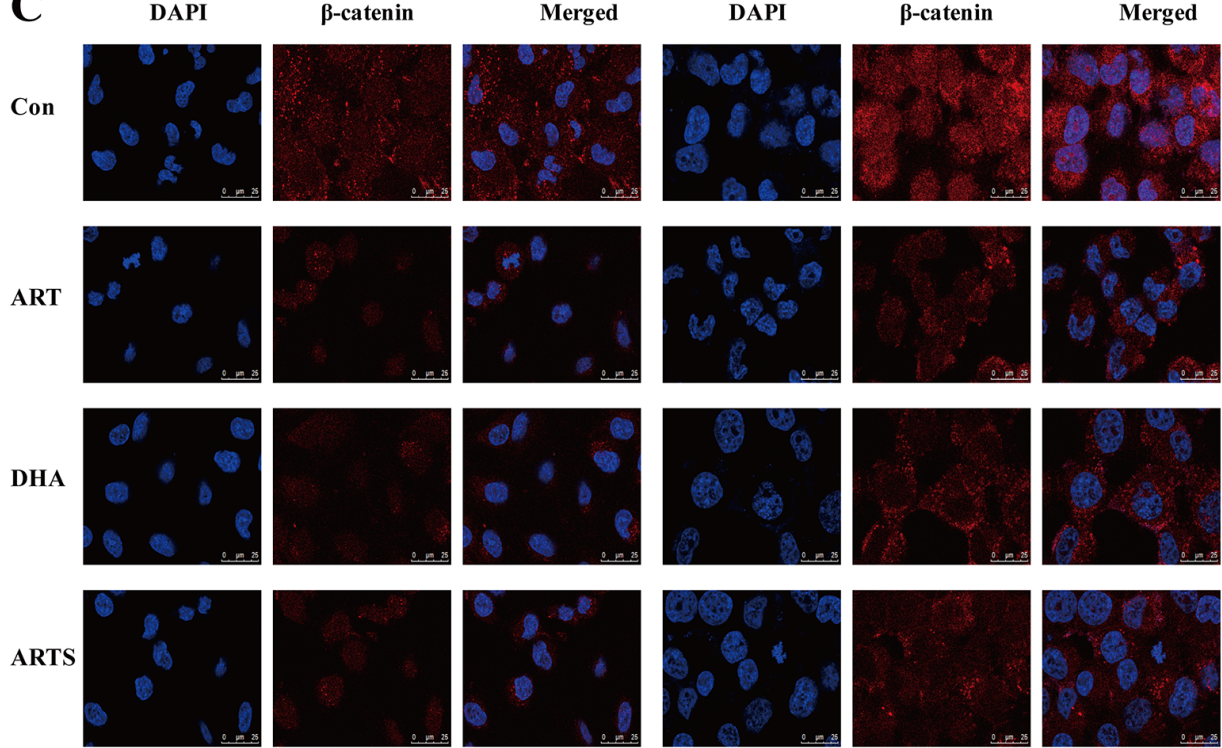

Figure 4: ART, DHA, and ARTS decreased Wnt5-a/b-triggered suppression of downstream proteins in vitro. A. A549 cells were harvested, and Western blot analysis was conducted for Wnt5-a/b, LRP6, Dvl2, and $\beta$-catenin. B. H1299 cells were harvested, and Western blot analysis was conducted for Wnt5-a/b, LRP6, Dvl2, and $\beta$-catenin. Data shown were the means \pm SD. $* * p<0.01, * * * p<0.001$, compared with the control group. C. Representative confocal imaging of nuclei (blue), $\beta$-catenin (red), and nuclei/ $\beta$-catenin double-stained cells at the level of $\beta$-catenin cytoplasm and nuclear localization after $48 \mathrm{~h}$ of ART, DHA, and ARTS treatments. Nuclei were counterstained with DAPI (scale bar: $25 \mu \mathrm{m}$ ). 
A
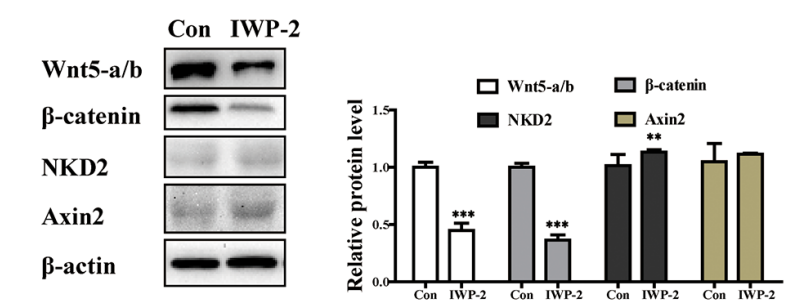

B
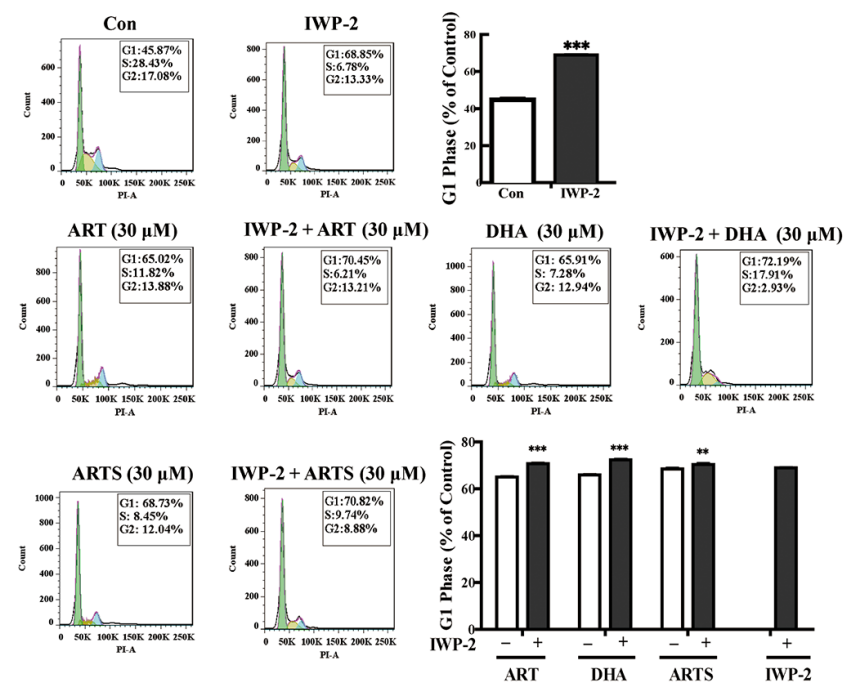

C

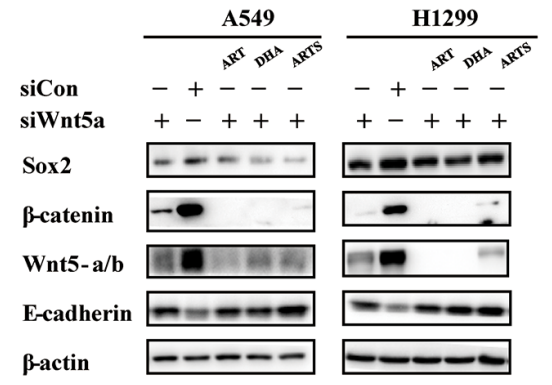

A549
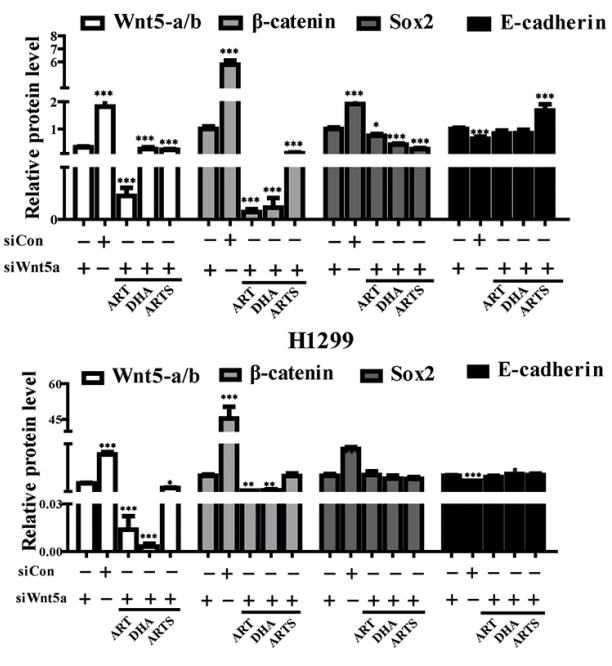

D
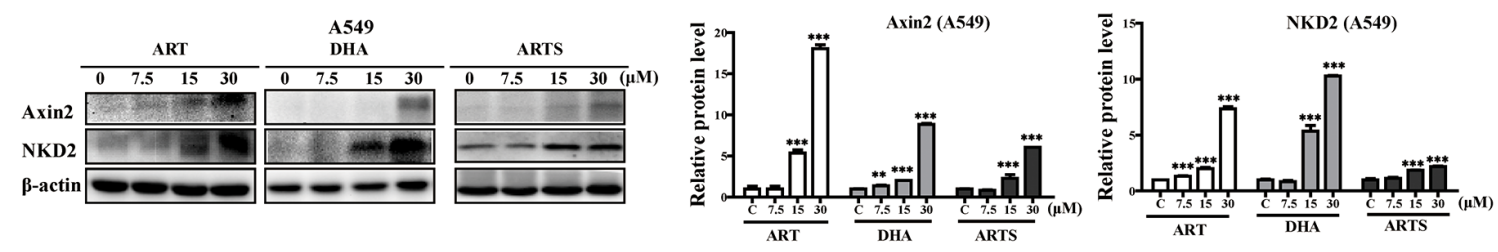

$\mathbf{E}$
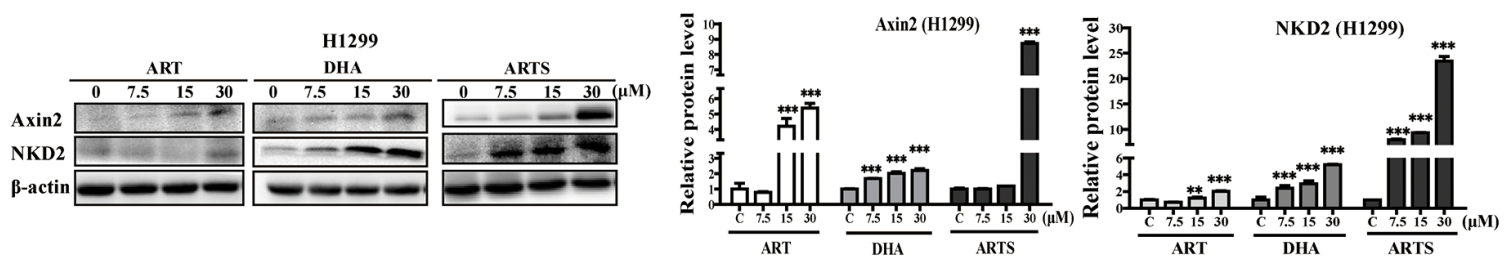

Figure 5: ART, DHA, and ARTS were partially dependent on Wnt/ $\boldsymbol{\beta}$-catenin signaling. A. A549 cells were harvested, and Western blot analysis was conducted for $\beta$-catenin, Wnt5-a/b, NKD2, and Axin2 with or without IWP-2. B. Percentage of A549 cells in the G1 phase with 0 and $30 \mu \mathrm{M}$ ART, DHA, and ARTS treatments accompanied with or without IWP-2. C. Cells were transfected with Wnt-5a siRNA or control siRNA before ART, DHA, and ARTS $(30 \mu \mathrm{M})$ treatments. Wnt5a, $\beta$-catenin, sox2, and E-cadherin protein levels were detected by Western blot analysis. D. A549 cells were harvested, and Western blot analysis was performed for Axin2 and NKD2. E. H1299 cells were harvested, and Western blot analysis was conducted for Axin2 and NKD2. The data represent mean $\pm \operatorname{SD} .{ }^{*} p<0.05, * * p<0.01$, $* * * p<0.001$, compared with the control group (Con, control).

staining was performed to confirm $\beta$-catenin expression in situ. Compared with the control group, $\beta$-catenin protein level was significantly decreased by approximately $50 \%$ in tumor tissues after ART, DHA, and ARTS treatments (Figure 6C). Furthermore, the expression levels of oct3/4, sox 2 , and nanog were respectively decreased by $5.10 \% \pm$
$0.25 \%(p<0.001), 19.30 \% \pm 0.19 \%(p<0.001)$, and $7.92 \%$ $\pm 0.15 \%(p<0.001)$ in ARTS treatment. By contrast, the inhibition effect of ART was less significant than those of ARTS and DHA. Similarly, ARTS and DHA remarkably decreased the expression of vimentin more than that observed in ART treatment (Figure 6D). Additionally, in 

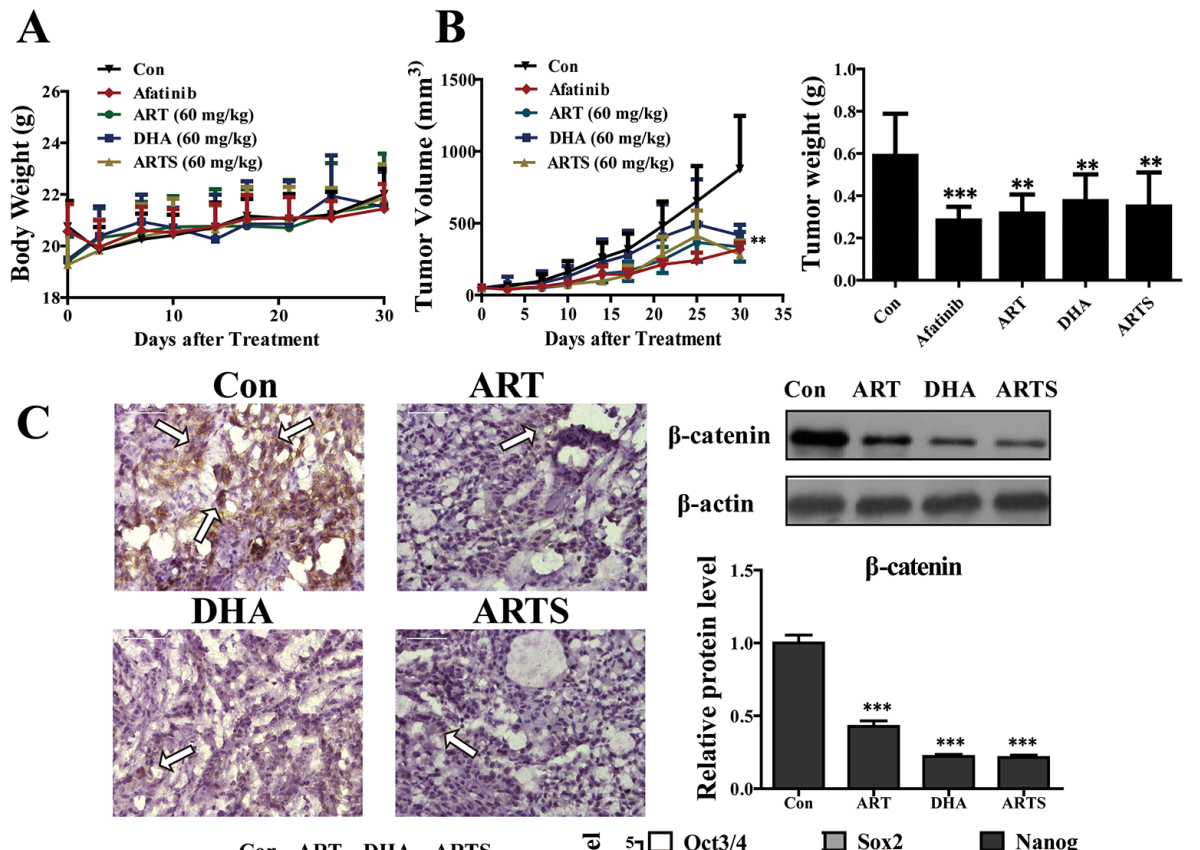

D
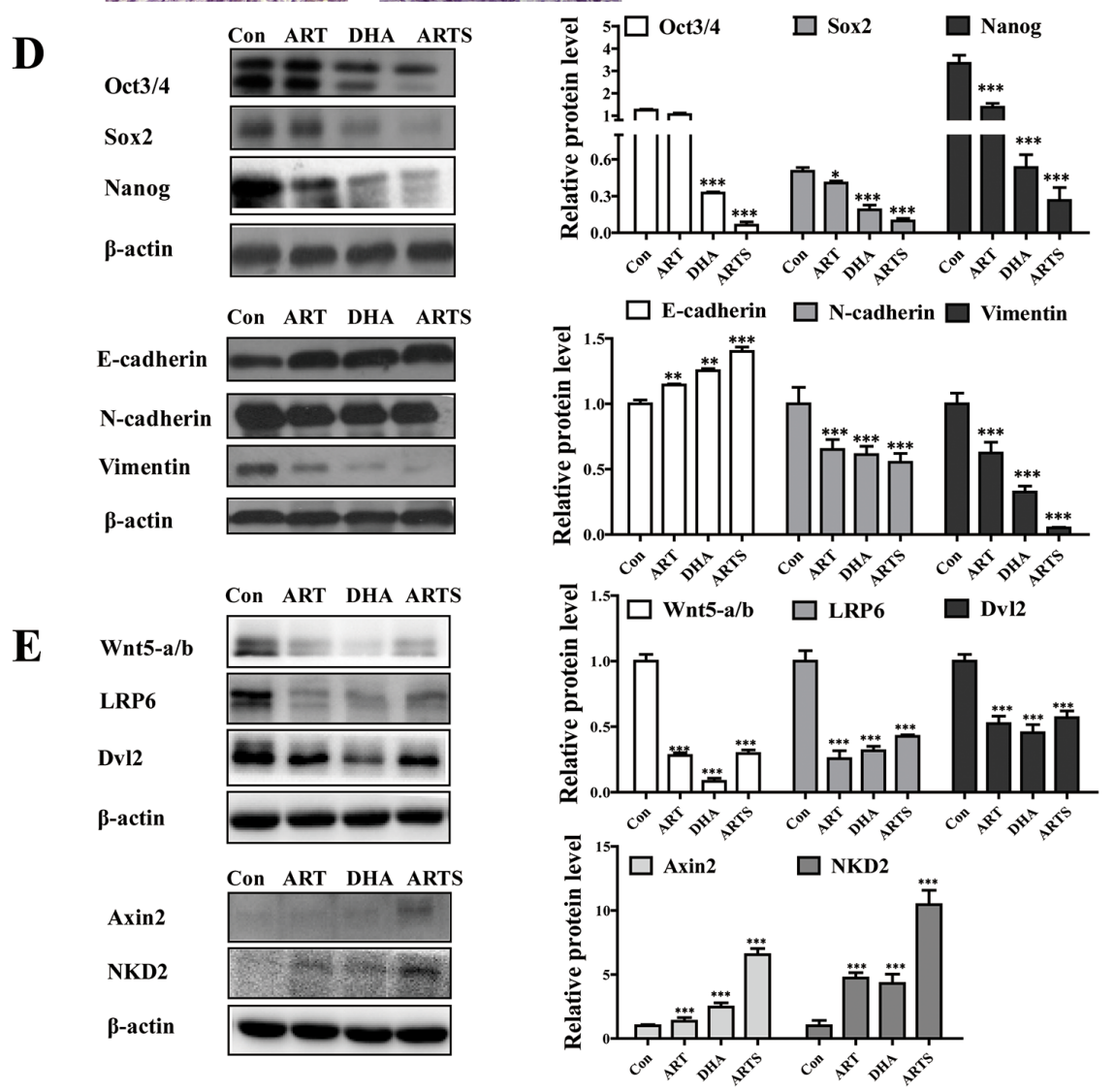

Figure 6: ART, DHA, and ARTS suppressed cell proliferation in A549 xenograft mice model by inhibiting Wnt//catenin, EMT and CSCs in vivo. A. Body weights of mice during the ART, DHA, and ARTS treatments. B. Tumor volume was measured during the experiment, and tumor weights of A549 xenografts were measured after four weeks of treatment. C. High magnification $(400 \times)$ of tumor sections treated with or without ART, DHA, and ARTS. A high signal corresponding to $\beta$-catenin protein in control group was detected (bar: $50 \mu \mathrm{m}$ ). Protein expression of $\beta$-catenin in lysates of tumor tissue from mouse was detected by Western blot. D. Protein expressions of markers of EMT and CSCs, namely, E-cadherin, N-cadherin, vimentin, nanog, sox2, and oct3/4 were detected by Western blot. E. Protein expressions of Wnt5-a/b, LRP6, Dvl2, Axin2, and NKD2 in lysates of tumor tissues from mice were detected by Western blot. Data shown were the means $\pm \mathrm{SD},{ }^{*} p<0.05,{ }^{* *} p<0.01,{ }^{* * *} p<0.001$, compared with the control group (Con, control). 
tumor tissues collected from A549 xenograft model, ART and its derivatives effectively reduced Wnt5-a/b, LRP6, and Dvl2 but significantly increased both NKD2 and Axin2 (Figure 6E; $p<0.001$ ).

\section{DISCUSSION}

For lung cancer patients in stages II and IIIa, surgical resection complemented with target therapeutics may achieve a good prognosis [38]. However, chemotherapies, act as a double-edged sword, could also simultaneously lead to numbers of side effects, such as diarrhea, headache, and ototoxicity when exert anti-tumor effectiveness [39, 40]. Research has demonstrated that ART has neurotoxicity and embryotoxicity in various species, although much more clinical studies and metaanalyses have found no serious side effects of ART, DHA, and ARTS $[41,42]$. While the toxicity of ART in mammal pneumonocytes also remains unconfirmed [43, 44]. According to our MTT results, among the three compounds, ART had the lowest cytotoxicity to lung tumor cells, whereas its metabolite DHA exerted highest (Figure 1A). More strikingly, compared with the cell viability suppression and cell cycle arrest observed in tumor cells, ART, DHA, and ARTS had minor toxicity on the growth of normal human bronchial epithelial cells, without even slight influencing the cell cycle of BEAS2B (Supplementary Figure S1). These results suggested that ART and its derivatives were potential excellent chemopreventive agents with almost no cytotoxicity to normal pneumonocytes.

Clinical studies have shown that although target therapeutic agents could prolong progression-free survival to 9.7 months, response rate varied due to individual differences [45]. For example, erlotinib, a sensitive EGFR tyrosine kinase inhibitor, could arouse only a $58 \%$ response in NSCLC patients [46]. By examining cell viabilities in lung carcinoma cells (A549) and nonsmall lung cancer cells (H1299), respectively, we found that regardless of histological clarification, ART, DHA, and ARTS may exert broad tumor suppressions in vitro. In both these two lung cancer cell lines, ART, DHA, and ARTS suppressed the proliferation of tumor cells by arresting their cycle in G1 phase and simultaneously decreasing cyclin D1 expression (Figures $1 \mathrm{~A}-1 \mathrm{C})$. The non-dose-dependent down-regulation of cyclin D1 may be ascribed to other regulators participated in G1 phase, such as cyclin E, cdk2, and cdk4 [47, 48].

Tumor-metastasis inhibition is a main criterion in determining whether a compound could be an effective chemopreventive agent [49]. Our data showed that ART, DHA, and ARTS significantly suppressed both cell migration and invasion in vitro either in A549 or H1299 cells (Figures 2A-2C). Furthermore, according to mechanism studies, the successful initiation of metastatic progression known as "metastatic colonization" is accomplished only by a minority of cancer cells, such as CSCs [16]. Thus, we evaluated the protein expression of the CSC markers nanog, sox2, and oct3/4 in the present work. As shown in Figure 3A and Supplementary Figure S2A, ART, DHA, and ARTS decreased the expression of these CSCs markers in lung cancer cells, even in tumor tissues in vivo (Figure 6D), suggesting an inhibition of these compounds on CSCs pluripotency. Simultaneously, the EMT markers N-cadherin and vimentin were markedly inhibited by ART, DHA, and ARTS treatments, whereas E-cadherin was significantly increased either in vitro (Figure 3B and Supplementary Figure S2B) or in vivo (Figure 6D). Therefore, with notable anti-metastasis effectiveness of ART and its derivatives strengthens their potentials as chemopreventive candidates.

To further explore the anti-tumor effectiveness of ART, DHA, and ARTS in vivo and also the implied risk of toxicity after biotransformation, A549 xenograft model was applied. Compared with the control group, without altering the body weight of mice, ART, DHA, and ARTS significantly decreased tumor volume and tumor weight (Figure 6A and 6B). Afatinib, an outstanding chemotherapeutic agent, not served as a positive control drug in our experiment, but only be chosen because of its oral administration regardless of its EGFR inhibitory effects. Subsequently, by staining tumor tissues, the protein expression of $\beta$-catenin decreased after ART, DHA, and ARTS treatments, indicating that $\beta$-catenin inactivation may be involved in their anti-tumor effects.

$\mathrm{Wnt} / \beta$-catenin pathway plays a critical role in every stage of cancer progression, including initiation, development, and metastasis [50, 51]. Furthermore, because of the potential to maintain cancer stem cells and EMT, it is regarded as an attractive target for cancer prevention [52]. In the present study, we observed that ART, DHA, and ARTS inhibited the expression level of Wnt5-a/b, down-regulated those of LRP6 and Dvl2, and subsequently reduced those of downstream genes mediated by $\beta$-catenin (i.e., nanog, sox 2 , oct $3 / 4$, and cyclin D1) in two lung cancer cell lines (Figure 4). Additionally, the inhibition of ART, DHA, and ARTS in Wnt/ $\beta$-catenin activation was also confirmed in tumor tissues collected from xenograft model in vivo (Figure 6E). Given the significant inhibition of $\mathrm{Wnt} / \beta$-catenin pathway in ART, DHA, and ARTS treatments, we further determined the molecular target of ART, DHA, and ARTS using Wnt5a siRNA as well as IWP-2, specific inhibitor of Wnt/ $\beta$-catenin pathway [53]. Compared with IWP-2, no significant difference in G1 arrest was observed in IWP2 combined with ART, DHA, and ARTS, respectively, indicating that $\mathrm{Wnt} / \beta$-catenin signaling pathway was probably the most vital pathway influenced by ART, DHA, and ARTS (Figure 5B). Moreover, Wnt5a silencing via siRNA did not fully abrogate the effect of ART, DHA, and ARTS on Wnt/ $\beta$-catenin pathway and downstream proteins, suggesting although very important, Wnt5a was 


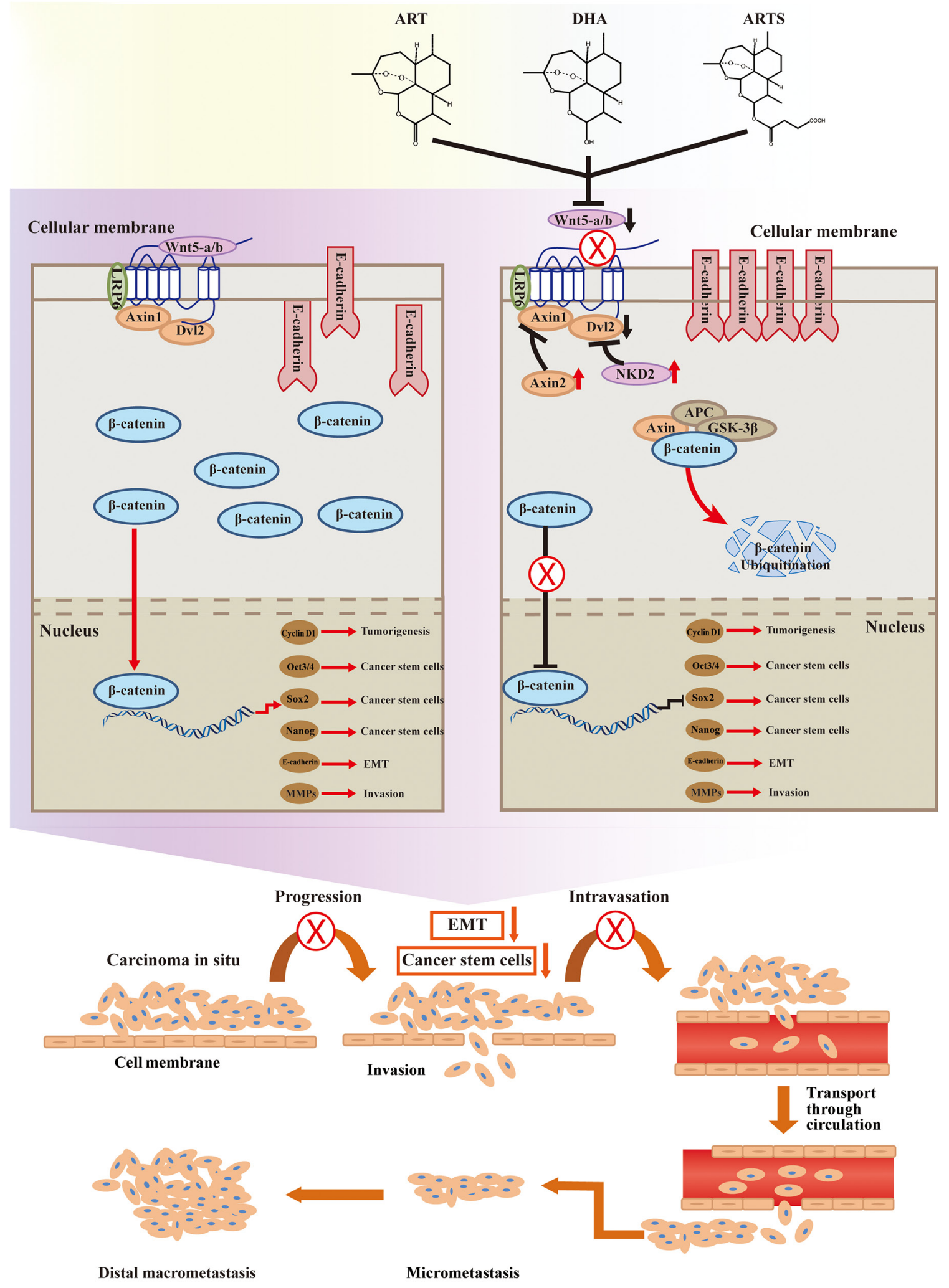

Figure 7: Inhibition of canonical Wnt/ק-catenin signaling. Canonical Wnt signaling was inhibited by ART, DHA, and ARTS. Besides inhibiting Wnt signaling through their interaction with Frizzled, secreted frizzled-related proteins may also inhibit the EMT. $\beta$-catenin can inhibit the transcription of CSCs and reduce the levels of cyclin D1. Axin2 and NKD2 are two negative regulators of Wnt/ $\beta$ catenin signaling. Axin2 and NKD2 expression was negative in resected NSCLC. (X, $\perp$, suppression of this progress. $\downarrow$, decrease of protein expression. $\uparrow$, increase of protein expression.) 
still not the only target for ART and its derivatives (Figure 5C). Other than that, Axin2 and NKD2, negative regulators in the canonical Wnt/ $\beta$-catenin signaling pathway $[9,54]$, were significantly increased as well by ART, DHA, and ARTS. Thus, apart from Wnt5a, negative mediators were also potential targets of ART, DHA, and ARTS (Figure 5D and $5 \mathrm{E}$ ). Schematic representation of targets, mediated by ART, DHA, and ARTS, in Wnt/ $\beta$-catenin pathway was shown in Figure 7.

Considering the irreversible drug resistance and cytotoxicity of chemotherapy, its benefits in the treatment of and life-quality improvement of early-stage lung-cancer patients remain controversial. By affecting multiple targets in Wnt/ $\beta$-catenin pathway, a developmental pathway influencing the clinical outcomes of early-diagnosed lung cancer patients, ART, DHA, and ARTS markedly suppressed lung tumorigenesis and exerted a perfect effect on inhibiting tumor metastasis. Therefore, ART and its derivatives are excellent chemopreventive candidates with high bioavailability, low toxicity, and significant anti-tumor efficacy [29]. Further studies should be conducted in chemical-induced lung cancer models such as benzopyrene (BaP)-mediated lung adenocarcinoma in $\mathrm{A} / \mathrm{J}$ mice to evaluate and confirm the chemopreventive effect of ART and its derivatives.

\section{MATERIALS AND METHODS}

\section{Chemicals and reagents}

ART, DHA, and ARTS were purchased from Dalian Meilun Biotech Co., Ltd (Dalian, China). IWP-2 and 3-(4, 5-dimethylthiazol-2-yl)-2, 5-diphenyltetrazolium bromide (MTT) were obtained from Sigma-Aldrich. Primary antibodies for LRP6, Axin2, Dv12, NKD2, and cyclin D1 were purchased from Cell Signaling Technology, Inc. And $\beta$-actin, oct3/4, nanog, sox2, E-cadherin, N-cadherin, and vimentin primary antibodies, secondary HRP-conjugated antibodies, Wnt5a siRNA and respective control siRNA were purchased from Santa Cruz Biotechnology. Primary antibodies for Wnt5a and $\beta$-catenin IHC staining were purchased from Abcam. Western blot detection reagents were obtained from Bio-Rad Laboratories. Amplite ${ }^{\mathrm{TM}}$ Universal Fluorimetric MMPs Activity Assay Kit was purchased from AAT Bioquest. Propidium iodide (PI) staining and matrigel were purchased from Biosciences.

\section{Cell lines}

The human lung cancer cells (A549 and H1299) and bronchial epithelial cells (BEAS-2B) were purchased from ATCC (Manassas, Virginia, USA). A549 and H1299 cells were cultured in RPMI-1640 medium, while BEAS-2B cells were maintained in BEGM medium supplemented with $10 \%$ FBS and $1 \%$ penicillin-streptomycin solution in a humidified atmosphere at $37{ }^{\circ} \mathrm{C}$ with $5 \% \mathrm{CO}_{2}$. And BEAS-2B was cultured in BEGM medium.

\section{Animals and xenograft model}

Investigation has been conducted in accordance with the ethical standards and according to the Declaration of Helsinki and according to national and international guidelines and has been approved by the authors' institutional review board.

Animal experiments, approved by the Guangzhou University of Chinese Medicine Animal Care and Use Committee (Guangzhou, China), have been conducted in accordance with the ethical standards and national guidelines. Female Balb/c-nude mice (4-6 weeks, 18$20 \mathrm{~g}$ ) were purchased from Laboratory Animal Center of Sun Yat-Sen University (Guangzhou, China), and then subjected to subcutaneously injection with A459 cells $\left(2 \times 10^{6}\right.$, suspended in PBS $)$ in each right flank. Tumor volume (TV) was defined based on two dimensions (L, long diameter; $\mathrm{W}$, wide diameter) measured by calipers, and calculated as formula: TV $\left(\mathrm{mm}^{3}\right)=\left(\mathrm{L} \times \mathrm{W}^{2}\right) / 2$. When the tumors reached a mean volume of $50 \mathrm{~mm}^{3}$, all mice were randomized into following groups: gavage control (sterilized coin oil), afatinib (5 mg/kg/d), ART (60 mg/ $\mathrm{kg} / \mathrm{d})$, DHA (60 mg/kg/d), and ARTS (60 mg/kg/d). Body weights were recorded twice a week. Treatments were administered orally five times a week for four weeks. At the end point, sacrificed the mice, and removed the tumors for following assays.

\section{Immunohistochemistry}

Tumor tissue specimens were fixed in neutral formalin and embedded in paraffin after collection from the sacrificed mice. Then tissue sections were cut and dewaxed, they were incubated with $0.01 \mathrm{M}$ natrium citricum for antigen retrieval. The slides were rinsed in phosphatebuffered saline and incubated overnight at $4{ }^{\circ} \mathrm{C}$ with diluted anti- $\beta$-catenin antibodies. Following steps were performed using the immunostaining kit (BOSTER Biological Technology) according to the manufacturer's instructions.

\section{MTT assay}

The MTT assay was used to evaluate the cell viability. $2 \times 10^{4}$ cells/ml of A549, H1299, and BEAS-2B cells were seeded into 96-well plates and cultured with different doses of ART, DHA, and ARTS for up to $48 \mathrm{~h}$. At the end of treatment, $0.5 \mathrm{mg} / \mathrm{ml}$ of MTT was added to the samples and incubated for $4 \mathrm{~h}$. Then the supernatants were discarded and coloured formazan crystals dissolved with $150 \mu \mathrm{l} /$ well of DMSO. Then OD values were read by using a microplate reader Victor X3 (PerkinElmer, Waltham, MA, USA) at $570 \mathrm{~nm}$. 


\section{Cell cycle analysis}

$1 \times 10^{6}$ cells $/ \mathrm{ml}$ of A549, H1299, and BEAS-2B were treated with vehicle or ART, DHA, and ARTS, for up to $48 \mathrm{~h}$. For cell cycle analysis, cells were harvested, fixed with $70 \%$ cold ethanol, incubated with RNase, and stained with propidium iodide. Cell cycle was detected by flow cytometry (BD Biosciences, San Diego, CA, USA) and analyzed by FlowJo v7.6 software.

\section{Wound healing assay}

Wound healing assay was conducted to examine the capacity of cell migration. Cells were treated with $0,7.5$, 15 , or $30 \mu$ M ART, DHA, and ARTS in RPMI 1640 media (1\% FBS). Photomicrographs of final wounds were taken at 24 and $48 \mathrm{~h}$. Photomicrographs of initial wounds were taken by DMI 300B Leica at $100 \times$ magnification. Initial and final wound sizes were measured using Image Pro Plus 6.0 software.

\section{Transwell assay}

Invasion analysis was performed by seeding A549 cells $\left(1 \times 10^{5}\right)$ treated with $7.5,15$, or $30 \mu \mathrm{M}$ ART, DHA, and ARTS into the upper chamber of a transwell apparatus coated with Matrigel and incubating the cells for $48 \mathrm{~h}$. Cells were stained with crystal violet. The non-invasive cells were scraped off with cotton swabs, whereas the invasive cells stained with crystal violet were dissolved in DMSO for measurement.

\section{Immunofluorescence}

Immunofluorescence was performed on A549 cells seeded on $15 \mathrm{~mm}$ confocal dish with $30 \mu \mathrm{M}$ ART, DHA, ARTS treating for $48 \mathrm{~h}$ and fixed in cold $4 \%$ paraformaldehyde. The confocal dishes were incubated with primary antibodies. Detection of the primary antibodies was performed using 1:500 Alexa Fluor ${ }^{\circledR}$ conjugated secondary antibodies. The images were captured and analyzed using Leica TCS SP8 confocal microscope. All images were acquired using an identical acquisition time for all samples.

\section{Wnt5a siRNA transfection}

A549 were plated into a 6-well plate with RPMI-1640 cell culture medium with 10\% FBS. siRNA transfection was performed according to the manufacturer's protocol. Seven hours post-transfection, cells were treated with different concentrations $(0,7.5,15$, and $30 \mu \mathrm{M}$ ) of ART, DHA, and ARTS for $48 \mathrm{~h}$. Cell lysates were analyzed for expression of Wnt5-a/b, $\beta$-catenin, Sox2, Oct $3 / 4$, N-cadherin, and E-cadherin proteins by Western blot analysis.

\section{Western blot}

Total proteins were extracted from cells after treatments. Protein samples were separated on $8 \%-12 \%$ SDS-polyacrylamide gel electrophoresis. Blots were immunostained with primary and secondary antibodies. $\beta$-actin served as a loading control. Other detailed procedures were followed according to the literature [20].

\section{MMPs activity assay}

MMPs activities were assessed using Amplite ${ }^{\mathrm{TM}}$ Universal Fluorimetric MMP Activity Assay Kit, according to standard protocols. In brief, 5,000 cells were seeded onto 24-well and then exposed to different concentrations $(0,7.5,15$, and $30 \mu \mathrm{M})$ of ART, DHA, and ARTS for $48 \mathrm{~h}$. Finally, $50 \mu \mathrm{L}$ of supernatants from each well was added to a 96-well plate for detection.

\section{Data analysis}

Data were expressed as the mean \pm standard deviation (SD) of at least three independent experiments. Statistical significance of the data was determined by oneway ANOVA using SPSS software. Statistical significance was considered at $p<0.05$.

\section{ACKNOWLEDGMENTS}

This work was supported by the grants of National Natural Science Foundation of China (81473410 and 81503466), Science and Technology Project of Guangzhou City (201509010004) and Guangdong Natural Science Foundation Province (2015AD030312012).

\section{CONFLICTS OF INTEREST}

The authors declare that they have no conflict of interest.

\section{REFERENCES}

1. Torre LA, Bray F, Siegel RL, Ferlay J, Lortet-Tieulent J and Jemal A. Global cancer statistics, 2012. CA Cancer J Clin. 2015; 65:87-108.

2. Jemal A, Bray F, Center MM, Ferlay J, Ward E and Forman D. Global cancer statistics. CA Cancer J Clin. 2011; 61:69-90.

3. Gridelli C, Rossi A and Maione P. The potential role of histone deacetylase inhibitors in the treatment of nonsmall-cell lung cancer. Critical reviews in oncology/ hematology. 2008; 68:29-36.

4. Stella GM, Luisetti M, Pozzi E and Comoglio PM. Oncogenes in non-small-cell lung cancer: emerging 
connections and novel therapeutic dynamics. The Lancet Respiratory medicine. 2013; 1:251-261.

5. Holohan C, Van Schaeybroeck S, Longley DB and Johnston PG. Cancer drug resistance: an evolving paradigm. Nature reviews Cancer. 2013; 13:714-726.

6. Surh YJ. Cancer chemoprevention with dietary phytochemicals. Nature reviews Cancer. 2003; 3:768-780.

7. Stewart DJ, Chang DW, Ye Y, Spitz M, Lu C, Shu X, Wampfler JA, Marks RS, Garces YI, Yang P and Wu X. Wnt signaling pathway pharmacogenetics in non-small cell lung cancer. The pharmacogenomics journal. 2014; $14: 509-522$

8. Liu D, Li L, Yang Y, Liu W and $\mathrm{Wu} \mathrm{J}$. The Axin2 rs2240308 polymorphism and susceptibility to lung cancer in a Chinese population. Tumour biology. 2014; 35:10987-10991.

9. Hu T, Li C, Cao Z, Van Raay TJ, Smith JG, Willert K, Solnica-Krezel L and Coffey RJ. Myristoylated Naked2 antagonizes Wnt-beta-catenin activity by degrading Dishevelled-1 at the plasma membrane. The Journal of biological chemistry. 2010; 285:13561-13568.

10. Kishida S, Yamamoto H, Hino S, Ikeda S, Kishida M and Kikuchi A. DIX domains of Dvl and axin are necessary for protein interactions and their ability to regulate betacatenin stability. Molecular and cellular biology. 1999; 19:4414-4422.

11. Bravo DT, Yang YL, Kuchenbecker K, Hung MS, Xu Z, Jablons DM and You L. Frizzled-8 receptor is activated by the Wnt-2 ligand in non-small cell lung cancer. BMC cancer. 2013; 13:316.

12. Wang C, Yang W, Yan HX, Luo T, Zhang J, Tang L, Wu FQ, Zhang HL, Yu LX, Zheng LY, Li YQ, Dong W, He YQ, Liu Q, Zou SS, Lin Y, et al. Hepatitis B virus X (HBx) induces tumorigenicity of hepatic progenitor cells in 3,5-diethoxycarbonyl-1,4-dihydrocollidine-treated HBx transgenic mice. Hepatology. 2012; 55:108-120.

13. You Z, Saims D, Chen S, Zhang Z, Guttridge DC, Guan KL, MacDougald OA, Brown AM, Evan G, Kitajewski $\mathrm{J}$ and Wang CY. Wnt signaling promotes oncogenic transformation by inhibiting c-Myc-induced apoptosis. The Journal of cell biology. 2002; 157:429-440.

14. Okita $\mathrm{K}$ and Yamanaka $\mathrm{S}$. Intracellular signaling pathways regulating pluripotency of embryonic stem cells. Current stem cell research \& therapy. 2006; 1:103-111.

15. Pan $G$ and Thomson JA. Nanog and transcriptional networks in embryonic stem cell pluripotency. Cell research. 2007; 17:42-49.

16. Malanchi I, Santamaria-Martinez A, Susanto E, Peng H, Lehr HA, Delaloye JF and Huelsken J. Interactions between cancer stem cells and their niche govern metastatic colonization. Nature. 2012; 481:85-89.

17. Subramaniam G, Campsteijn $\mathrm{C}$ and Thompson EM. Co-expressed Cyclin D variants cooperate to regulate proliferation of germline nuclei in a syncytium. Cell cycle. 2015; 14:2129-2141.

18. Douchi D, Ohtsuka H, Ariake K, Masuda K, Kawasaki S, Kawaguchi K, Fukase K, Oikawa M, Motoi F, Naitoh T, Katayose Y, Egawa S and Unno M. Silencing of LRRFIP1 reverses the epithelial-mesenchymal transition via inhibition of the Wnt/beta-catenin signaling pathway. Cancer letters. 2015; 365:132-140.

19. Holcombe RF, Martinez M, Planutis K and Planutiene M. Effects of a grape-supplemented diet on proliferation and Wnt signaling in the colonic mucosa are greatest for those over age 50 and with high arginine consumption. Nutrition journal. 2015; 14:62.

20. Bi X, Zhao Y, Fang W and Yang W. Anticancer activity of Panax notoginseng extract 20(S)-25-OCH3-PPD: Targetting beta-catenin signalling. Clinical and experimental pharmacology \& physiology. 2009; 36:1074-1078.

21. Zhao Y, Yao J, Wu XP, Zhao L, Zhou YX, Zhang Y, You QD, Guo QL and Lu N. Wogonin suppresses human alveolar adenocarcinoma cell A549 migration in inflammatory microenvironment by modulating the IL-6/ STAT3 signaling pathway. Molecular carcinogenesis. 2015; 54 Suppl 1:E81-93.

22. Dragnev K, You M, Wang Y and Lubet R. Lung cancer chemoprevention: difficulties, promise and potential agents? Expert opinion on investigational drugs. 2013; 22:35-47.

23. Qian X, Song JM, Melkamu T, Upadhyaya P and Kassie F. Chemoprevention of lung tumorigenesis by intranasally administered diindolylmethane in $\mathrm{A} / \mathrm{J}$ mice. Carcinogenesis. 2013; 34:841-849.

24. Efferth T, Dunstan H, Sauerbrey A, Miyachi H and Chitambar CR. The anti-malarial artesunate is also active against cancer. International journal of oncology. 2001; 18:767-773.

25. Eastman RT and Fidock DA. Artemisinin-based combination therapies: a vital tool in efforts to eliminate malaria. Nature reviews Microbiology. 2009; 7:864-874.

26. Dondorp AM, Fairhurst RM, Slutsker L, Macarthur JR, Breman JG, Guerin PJ, Wellems TE, Ringwald P, Newman $\mathrm{RD}$ and Plowe CV. The threat of artemisinin-resistant malaria. The New England journal of medicine. 2011; 365:1073-1075.

27. Kakuru A, Jagannathan $P$, Muhindo MK, Natureeba $P$, Awori P, Nakalembe M, Opira B, Olwoch P, Ategeka J, Nayebare P, Clark TD, Feeney ME, Charlebois ED, Rizzuto G, Muehlenbachs A, Havlir DV, et al. Dihydroartemisinin-Piperaquine for the Prevention of Malaria in Pregnancy. The New England journal of medicine. 2016; 374:928-939.

28. Nji AM, Ali IM, Moyeh MN, Ngongang EO, Ekollo AM, Chedjou JP, Ndikum VN, Evehe MS, Froeschl G, Heumann C, Mansmann U, Ogundahunsi O and Mbacham WF. Randomized non-inferiority and safety 
trial of dihydroartemisin-piperaquine and artesunateamodiaquine versus artemether-lumefantrine in the treatment of uncomplicated Plasmodium falciparum malaria in Cameroonian children. Malaria journal. 2015; $14: 27$.

29. Karunajeewa HA, Manning L, Mueller I, Ilett KF and Davis TM. Rectal administration of artemisinin derivatives for the treatment of malaria. Jama. 2007; 297:2381-2390.

30. Batty KT, Davis TM, Thu LT, Binh TQ, Anh TK and Ilett KF. Selective high-performance liquid chromatographic determination of artesunate and alpha- and betadihydroartemisinin in patients with falciparum malaria. Journal of chromatography B, Biomedical applications. 1996; 677:345-350.

31. Titulaer HA, Zuidema J, Kager PA, Wetsteyn JC, Lugt CB and Merkus FW. The pharmacokinetics of artemisinin after oral, intramuscular and rectal administration to volunteers. The Journal of pharmacy and pharmacology. 1990; 42:810-813.

32. Morris CA, Duparc S, Borghini-Fuhrer I, Jung D, Shin CS and Fleckenstein L. Review of the clinical pharmacokinetics of artesunate and its active metabolite dihydroartemisinin following intravenous, intramuscular, oral or rectal administration. Malaria journal. 2011; 10:263.

33. Chen T, Li M, Zhang R and Wang H. Dihydroartemisinin induces apoptosis and sensitizes human ovarian cancer cells to carboplatin therapy. Journal of cellular and molecular medicine. 2009; 13:1358-1370.

34. Wang SJ, Gao Y, Chen H, Kong R, Jiang HC, Pan SH, Xue DB, Bai XW and Sun B. Dihydroartemisinin inactivates NF-kappaB and potentiates the anti-tumor effect of gemcitabine on pancreatic cancer both in vitro and in vivo. Cancer letters. 2010; 293:99-108.

35. Gomes MF, Faiz MA, Gyapong JO, Warsame M, Agbenyega T, Babiker A, Baiden F, Yunus EB, Binka F, Clerk C, Folb P, Hassan R, Hossain MA, Kimbute O, Kitua A, Krishna S, et al. Pre-referral rectal artesunate to prevent death and disability in severe malaria: a placebocontrolled trial. Lancet. 2009; 373:557-566.

36. Rasheed SA, Efferth T, Asangani IA and Allgayer H. First evidence that the antimalarial drug artesunate inhibits invasion and in vivo metastasis in lung cancer by targeting essential extracellular proteases. International journal of cancer. 2010; 127:1475-1485.

37. Odaka Y, Xu B, Luo Y, Shen T, Shang C, Wu Y, Zhou H and Huang S. Dihydroartemisinin inhibits the mammalian target of rapamycin-mediated signaling pathways in tumor cells. Carcinogenesis. 2014; 35:192-200.

38. Field JK, Oudkerk M, Pedersen JH and Duffy SW. Prospects for population screening and diagnosis of lung cancer. Lancet. 2013; 382:732-741.

39. Schramm A, De Gregorio N, Widschwendter P, Fink $\mathrm{V}$ and Huober J. Targeted Therapies in HER2-Positive Breast Cancer - a Systematic Review. Breast care (Basel, Switzerland). 2015; 10:173-178.
40. Kramer R, Bielawski J, Kistner-Griffin E, Othman A, Alecu I, Ernst D, Kornhauser D, Hornemann T and Spassieva S. Neurotoxic 1-deoxysphingolipids and paclitaxelinduced peripheral neuropathy. FASEB journal. 2015; 29:4461-4472.

41. Das AK. Anticancer Effect of AntiMalarial Artemisinin Compounds. Annals of medical and health sciences research. 2015; 5:93-102.

42. Gordi T and Lepist EI. Artemisinin derivatives: toxic for laboratory animals, safe for humans? Toxicology letters. 2004; 147:99-107.

43. Toovey S. Safety of artemisinin antimalarials. Clinical infectious diseases. 2006; 42:1214-1215.

44. Clark RL. Embryotoxicity of the artemisinin antimalarials and potential consequences for use in women in the first trimester. Reproductive toxicology. 2009; 28:285-296.

45. Rosell R, Carcereny E, Gervais R, Vergnenegre A, Massuti B, Felip E, Palmero R, Garcia-Gomez R, Pallares C, Sanchez JM, Porta R, Cobo M, Garrido P, Longo F, Moran $\mathrm{T}$, Insa A, et al. Erlotinib versus standard chemotherapy as first-line treatment for European patients with advanced EGFR mutation-positive non-small-cell lung cancer (EURTAC): a multicentre, open-label, randomised phase 3 trial. The Lancet Oncology. 2012; 13:239-246.

46. Rosell R, Bivona TG and Karachaliou N. Genetics and biomarkers in personalisation of lung cancer treatment. Lancet. 2013; 382:720-731.

47. Camarillo JM, Rose KL, Galligan JJ, Xu S and Marnett LJ. Covalent Modification of CDK2 by 4-Hydroxynonenal as a Mechanism of Inhibition of Cell Cycle Progression. Chemical research in toxicology. 2016; 29:323-332.

48. Roskoski R, Jr. Cyclin-dependent protein kinase inhibitors including palbociclib as anticancer drugs. Pharmacological research. 2016.

49. Szabo E, Mao JT, Lam S, Reid ME and Keith RL. Chemoprevention of lung cancer: Diagnosis and management of lung cancer, 3rd ed: American College of Chest Physicians evidence-based clinical practice guidelines. Chest. 2013; 143:e40S-60S.

50. Hubaux R, Thu KL and Lam WL. Re: the Wnt signaling pathway in non-small cell lung cancer. Journal of the National Cancer Institute. 2014; 106.

51. Peng YY, He YH, Chen C, Xu T, Li L, Ni MM, Meng $\mathrm{XM}$, Huang $\mathrm{C}$ and Li J. NLRC5 regulates cell proliferation, migration and invasion in hepatocellular carcinoma by targeting the Wnt/beta-catenin signaling pathway. Cancer letters. 2016.

52. Bartis D, Csongei V, Weich A, Kiss E, Barko S, Kovacs T, Avdicevic M, D'Souza VK, Rapp J, Kvell K, Jakab L, Nyitrai M, Molnar TF, Thickett DR, Laszlo T and Pongracz JE. Down-regulation of canonical and up-regulation of non-canonical Wnt signalling in the carcinogenic process of squamous cell lung carcinoma. PloS one. 2013; 8:e57393. 
53. Xu H, Tsang KS, Wang Y, Chan JC, Xu G and Gao WQ. Unfolded protein response is required for the definitive endodermal specification of mouse embryonic stem cells via Smad2 and beta-catenin signaling. The Journal of biological chemistry. 2014; 289:26290-26301.
54. Bowman AN, van Amerongen R, Palmer TD and Nusse R. Lineage tracing with Axin2 reveals distinct developmental and adult populations of Wnt/beta-catenin-responsive neural stem cells. Proceedings of the National Academy of Sciences of the United States of America. 2013; 110:7324-7329. 